TRANSACTIONS OF THE

AMERICAN MATHEMATICAL SOCIETY

Volume 186, December 1973

\title{
THE VEECH STRUCTURE THEOREM
}

\author{
BY \\ ROBERT ELLIS( $\left.{ }^{1}\right)$
}

ABSTRACT. The main result is the proof of the Veech structure theorem for point-distal flows without the assumption that the distal points form a residual set. This allows one to conclude that, in the case of metrizable flows, if there is one distal point then there is a residual set of such points.

0. Introduction. In [3] Veech proves a structure theorem for point-distal transformation groups $(X, T)$ with compact metric phase space $X$ in which the set of distal points is residual. (See paragraph 1 for definitions.) The purpose of this paper is to give an alternative proof of this theorem without this latter assumption. It then follows as Veech pointed out that if the compact metric minimal transformation group $(X, T)$ has one distal point, then it has a residual set of distal points.

The essential step in the proof of the structure theorem is the insertion of a transformation group $(Z, T)$ between two transformation groups $(X, T)$ and $(Y, T)$. To be more specific: if $\pi:(X, T) \rightarrow(Y, T)$ be a homomorphism, then under certain conditions (see Theorem 7.1 of [3]) there exist a nontrivial isometric extension $\Psi:(Z, T) \rightarrow(Y, T)$ and a homomorphism $\phi:(X, T) \rightarrow(Z, T)$ such that the following diagram is commutative.

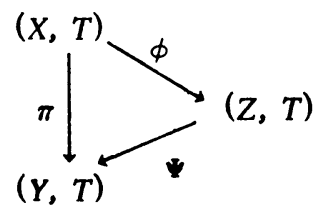

The conditions as stated by Veech involved the notion of point-distal directly. Recently Bronstein [1] replaced these by requiring that the almost periodic points of the relation, $R(X, Y)=\left\{\left(x_{1}, x_{2}\right) \mid x_{1}, x_{2} \in X\right.$ and $\left.\pi\left(x_{1}\right)=\pi\left(x_{2}\right)\right\}$ be dense in $R(X, Y)$. Using this condition he was able to give a direct proof of Veech's

Received by the editors June 5, 1972 and, in revised form, March 5, 1973.

AMS (MOS) subject classifications (1970). Primary 54H20; Secondary 54H15.

Key words and phrases. Distal, proximal, point-distal extension, almost automorphic, regionally proximal, equicontinuous structure relation.

(1) Research supported by NSF Grant GP-8691.

Copyright $\odot$ 1974, American Mathematical Society 
theorem. Indeed he also obtained the "relativized" version of the theorem. However, this proof also requires the assumption that there be a residual set of distal points. In this paper I replace Bronstein's condition by a weaker one involving the groups of the various minimal sets involved (see 2.1 below) and also obtain the relativized version of the theorem again without any assumption on the size of the set of "Y-distal-points" other than that it be nonvacuous.

Finally I show that the condition that $X$ be metrizable can be weakened to the condition that $X$ be quasi-separable, i.e. that $X$ have sufficiently many metrizable factors.

1. Notation and definitions. The main tools used in this paper are the algebraic techniques developed in [2] and thus a brief review of the notation used therein will be useful.

Throughout this work $T$ will denote a fixed group provided with the discrete topology, $\beta T$ its Stone-Čech compactification, and $M$ a fixed minimal subset of $\beta T$. The set $M$ is provided with a semigroup structure such that $M v$ is a group for every idempotent $v$ in $M$. One such idempotent, $u$ is fixed and the corresponding group, $M u$ is denoted by $G$.

To each $T$-subalgebra $\mathcal{Q}$ of $\mathcal{C}(\beta T)$ there corresponds canonically a transformation group $(|\mathfrak{Q}|, T)$ with compact $T_{2}$ phase space $|\mathfrak{Q}|$ such that $\mathfrak{Q} \cong \mathfrak{C}(|\mathfrak{Q}|)$. If $\mathfrak{Q} \subset a(u)=\{f \mid f \in \mathcal{C}(\beta T), f u=f\}$, then $(|\mathfrak{Q}|, T)$ is minimal. Moreover if $(X, T)$ is minimal, then there exists a $T$-subalgebra, $\mathfrak{Q}$ with $\mathfrak{Q} \subset a(u)$ and $(X, T) \cong(|\mathfrak{A}|, T)$. Moreover if $\pi: X \rightarrow Y$ is a homomorphism and $(Y, T) \cong(|\mathfrak{F}|, T)$ then $\pi$ goes over to the restriction map of $|\mathfrak{Q}|$ onto $|\mathfrak{F}|$; i.e., the map induced by the inclusion $\mathfrak{F} \subset \mathfrak{Q}$. Thus the situation discussed in the preceding paragraph may be translated into algebraic terms.

Henceforth $\mathfrak{F}$ and $\mathfrak{Q}$ will denote fixed $T$-subalgebras of $\mathcal{C}(\beta T)$ with $\mathcal{F} \subset \mathfrak{Q} \subset$ $a(u)$. In algebraic terms the essential step discussed in $\$ 0$ becomes the interpolation of a nontrivial isometric, or more generally an almost periodic, extension of $\mathcal{F}$ between $\mathcal{F}$ and $\mathfrak{Q}$. The largest such extension is just $\mathfrak{Q} \cap \mathfrak{F}^{\#}$, where $\mathfrak{F}^{\#}$ is the subset of $\mathcal{C}(\beta T)$ consisting of those functions which are almost periodic over $\mathcal{F}$ (see 14.3 and 14.21 of [2]). The problem then reduces to finding conditions on $\mathcal{F}$ and $\mathfrak{Q}$ which ensure that $\mathcal{F} \neq \mathfrak{Q} \cap \mathcal{F}^{\#}$. Another way of phrasing the condition $\mathfrak{F} \neq \mathfrak{Q} \cap \mathfrak{F}^{\#}$ is $R(\mathfrak{Q}: \mathcal{F}) \neq R\left(\mathbb{Q}: \mathfrak{Q} \cap \mathfrak{F}^{\#}\right)$. Here $R(\mathcal{K}: \mathfrak{L})=\{(x, y)|x, y \in| \mathcal{K} \mid$,

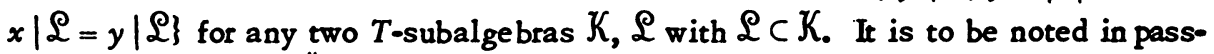
ing that $R\left(\mathfrak{G}: \mathfrak{Q} \cap \mathfrak{F}^{\#}\right)$ is just the relative (to $\left.|\mathfrak{F}|\right)$ equicontinuous structure relation on $|\mathfrak{A}|$.

In order to define the various notions mentioned in $\$ 0$ it is necessary to introduce the relativized proximal and regionally proximal relations, $P(\mathfrak{G}: \mathfrak{F})$ and 
$Q(\mathfrak{Q}: \mathcal{F})$ respectively. Here $P(\mathfrak{Q}: \mathcal{F})=\bigcap\{\eta T \cap R(\mathfrak{Q}: \mathcal{F}) \mid \eta \in \mathcal{U}\}$ and $Q(\mathfrak{Q}: \mathcal{F})=$ $\bigcap\{c l s(\eta T \cap R(\mathfrak{Q}: \mathcal{F})) \mid \eta \in \mathcal{U}\}$ where $\mathcal{U}$ is the uniformity on $|\mathfrak{Q}|$.

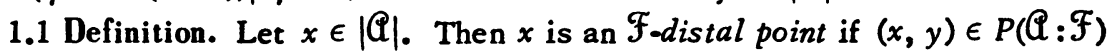
implies that $x=y \quad(y \in|\mathfrak{Q}|)$. The transformation group $(|\mathfrak{Q}|, T)$ is $\mathcal{F}_{\text {-point-distal }}$ if there exists an $\mathcal{F}_{\text {-distal point in }}|\mathfrak{Q}|$. Notice that if $x$ is an $\mathcal{F}_{\text {-distal point then }}$ so is $x t(t \in T)$.

1.2 Definition. The transformation group $(|\mathfrak{Q}|, T)$ is an almost automorpbic extension of $(|\mathfrak{F}|, T)$ if there exists $x \in|\mathfrak{Q}|$ such that $(y, x) \in R(\mathfrak{Q}: \mathcal{F})$ if and only if $y=x(y \in|\mathfrak{Q}|)$; i.e. the fiber over $\pi(x)$ consists only of the single point $x$; where $\pi$ is the canonical homomorphism of $(|\mathfrak{Q}|, T)$ onto $(|\mathfrak{F}|, T)$; i.e. the map induced by the inclusion $\mathcal{F} \subset \mathfrak{Q}$.

The principal result of this paper as it applies to the Veech theory may now be stated.

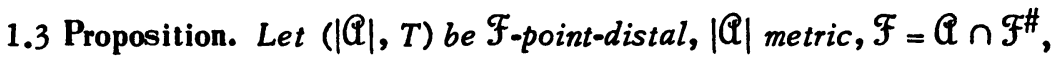
and the canonical map $\pi:|\mathfrak{Q}| \rightarrow|\mathfrak{F}|$ open. Then $\mathfrak{Q}=\mathscr{F}$.

(In other words, if $\mathfrak{Q} \neq \mathcal{F}$ and $\pi$ is open then $\mathcal{F} \neq \mathfrak{Q} \cap \mathcal{F}^{\#}$ and we can insert a nontrivial almost periodic extension of $\mathcal{F}$ between $\mathcal{F}$ and $\left.\mathfrak{Q}_{\text {.) }}\right)$

I shall not prove 1.3 directly but rather I shall prove a more general proposition from which 1.3 follows. In order to discuss the latter it is necessary to consider the group $H(F, \mathfrak{Q})$.

Let $F$ and $A$ denote the groups of $\mathcal{F}$ and $\mathfrak{Q}_{\text {respectively. Recall that every }}$ $T$-subalgebra, $\mathcal{K}$ of $\mathcal{C}(\beta T)$ induces a so-called r-topology, $\tau(\mathcal{K})$ on $G$ (see [2, p. 102]). Since $F \subset G, r(\mathcal{K})$ induces a topology on $F$. Then $H(F, \mathfrak{Q})=$ $\bigcap\left\{c 1 s s_{Q} V \mid V \in \Re_{Q}(F)\right\}$ where $\pi_{Q}(F)$ is the set of $r(\mathfrak{Q})$-neighborhoods of $u$ in $F$ (i.e. $V \in \pi_{G}(F)$ if and only if $V=W \cap F$ for some $\tau(\mathcal{Q})$-neighborhood $W$ of $u$ ) and "cls $Q$ " denotes closure with respect to the $r(\mathfrak{Q})$ topology on $G$.

By 14.16 of [2] the group of $\mathfrak{Q} \cap \mathcal{F}^{\#}$ is just $A F^{\#}$. Thus $\mathfrak{Q} \cap \mathcal{F}^{\#}=\mathcal{F}$ implies that $A F^{\#}=F$. Since $\mathfrak{Q} \cap \mathcal{F}^{\#} \subset \mathfrak{Q}, r\left(\mathbb{Q} \cap \mathcal{F}^{\#}\right) \subset \tau(\mathfrak{Q})$, whence $H(F, \mathfrak{Q}) \subset$ $H\left(F, \mathfrak{Q} \cap \mathfrak{F}^{\#}\right)$. By 14.15 of $[2] H\left(F, \mathfrak{Q} \cap \mathcal{F}^{\#}\right)=A F^{\#}$. Hence if $\mathfrak{Q} \cap \mathcal{F}^{\#}=\mathcal{F}$, $H(F, \mathfrak{Q})=F$.

The main idea of the paper is to show (under the assumptions of 1.3) first that $H(F, \mathfrak{Q})=F$, then that this implies $A=F$, and finally to conclude that $\mathfrak{Q}=\mathfrak{F}$.

The principal result of this paper is

1.4 Proposition. Let $H(F: \mathfrak{Q})=F$ and let $P(\mathscr{Q}: \mathcal{F})=\bigcap\left\{\eta_{i} T \cap R(\mathbb{Q}: \mathcal{F}) \mid i \in I\right\}$ where $\left(\eta_{i} \mid i \in I\right)$ is a countable set of indices of $|\mathfrak{Q}|$. Then there exists an idempotent $w$ in $M$ such that $w \mid \mathcal{F}$ is the identity and the set $\{p \mid p \in \overline{F w}$, $(p|\mathfrak{Q}, w| \mathfrak{Q}) \in P(\mathbb{Q}: \mathcal{F})\}$ is a residual subset of $\bar{F} w$. (Here $\bar{L}$ denotes the closure of $L$ with respect to the usual topology on $\beta T$, for all subsets $L$ of $\beta T$.) 
In this paper 1.4 is the essential tool used in proving the Veech structure theorem. However it has application to a wide range of problems which I hope to discuss in future papers.

2. The structure of the paper. In $\$ 3$ the structure of $H(F, \mathfrak{Q})$ is examined more closely. It is shown that $H(F, \mathfrak{Q})=\bigcap\left\{c l s_{\tau} V \mid V \in \Re_{\mathbb{Q}}(F)\right\}$, i.e. we may intersect the r-closures of neighborhoods of $u$ rather than $r(\mathbb{Q})$ closures of such neighborhoods to obtain $H(F, \mathfrak{Q})$. This characterization of $H(F, \mathfrak{Q})$ allows me to conclude that it is a ( $r$-closed) subgroup of $F$.

The above results are used in $\$ 4$ to prove Proposition 1.4 and in $\$ 5$ to prove

2.1 Proposition. Statements 1, 2, 3 are pairwise equivalent and they imply statements 4 and 5.

1. $H(F, \mathbb{Q}) \supset F^{\#}$.

2. $H(F, \mathfrak{Q})=A F^{\#}$.

3. $H(F, \mathfrak{Q})=\{\alpha \mid a \in F,(\alpha|\mathfrak{Q}, u| \mathfrak{Q}) \in Q(\mathfrak{Q}: \mathcal{F})\}$.

4. $R\left(\mathbb{Q}: \mathfrak{Q} \cap \mathfrak{F}^{\#}\right)=P(\mathbb{Q}: \mathfrak{F}) Q(\mathfrak{Q}: \mathcal{F})$.

5. $R\left(\mathfrak{Q}: \mathfrak{Q} \cap \mathfrak{F}^{\#}\right)=\{(x, y) \mid(x v, y v) \in Q(\mathbb{Q}: \mathfrak{F})$ for all minimal idempotents $v\}$ - $\cap R(\mathfrak{Q}: \mathfrak{F})$.

$\$ 6$ is devoted to proving that if the almost periodic points of $R(\mathfrak{Q}: \mathcal{F})$ are dense in $R(\mathfrak{Q}: \mathcal{F})$, then 3 of 2.1 holds. Then Bronstein's result [1] is recovered by 2.1 .

In $\$ 7$ the various results obtained are put together to obtain 1.3 and the Veech structure theorem. In proving the latter use is made of some of the results of [3]. Since these were obtained only for metrizable phase spaces they must be extended in order to prove the structure theorem for more general transformation groups. The subsequent section is devoted to this task.

3. The structure of $H(F, \mathbb{Q})$. For expository reasons it will be convenient to abstract the situation being considered. Thus in this section $K$ will denote a group provided with two topologies $\mathfrak{T}$ and $\mathfrak{S}$ satisfying the following conditions:

(i) $\mathfrak{T} \subset \mathcal{S}$.

(ii) $(K, \delta)$ is compact, $T_{1}$.

(iii) The maps $g \rightarrow g a:(K, \delta) \rightarrow(K, \delta)$ and $g \rightarrow a g:(K, \delta) \rightarrow(K, \delta)$ are continuous ( $a \in K$ ).

(iv) The maps $g \rightarrow g a:(K, \mathcal{T}) \rightarrow(K, \mathcal{T})$ are continuous $(g \in K)$.

Set $H(K, \mathcal{T})=\bigcap\left\{c l s_{g} V \mid V \in \Re_{g}\right\}$ and $L=\bigcap\left\{c l s_{\delta} V \mid V \in \Re_{g}\right\}$ where $\Re_{\delta}$ and $r_{g}$ are the neighborhoods of the identity $e$ with respect to the topologies $\mathcal{S}$ and $\mathfrak{J}$ respectively.

3.1 Lemma. $L$ is a subgroup of $K$. 


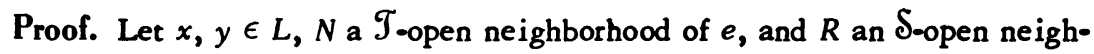
borhood of $e$. Then $y \in \operatorname{cls}_{\delta} N$ implies that $y R \cap N \neq \varnothing$ (by (iii)). Let $r \in R$ with $y r \in N$. Then by (iv) there exists $S \in \Re_{g}$ with $S_{y r} \subset N$. Hence (cls $S$ ) yr $\subset$ $\operatorname{cls}_{\delta}(N)$ (iii) and so $x y r \in \operatorname{cls}_{\delta}(N)$. Thus $x y R \cap \operatorname{cls} \delta N \neq \varnothing$, whence $x y R \cap N \neq \varnothing$ (iii). Since $R$ and $N$ were arbitrary, $x y \in L$. Lemma 3.1 now follows from $[2,2.11]$, (ii), and (iii).

\subsection{Lemma. $L=H(K, \mathcal{J})$.}

Proof. Since $\mathcal{T} \subset \mathcal{S}, \operatorname{cls}_{\mathcal{\delta}} N \subset \operatorname{cls}_{\tau} N\left(N \in \eta_{\tau}\right)$.

Now let $b \in H(K, \mathcal{T})$ and $N, R \mathcal{T}$-open neighborhoods of $e$. Then $N b \cap R \neq 0$

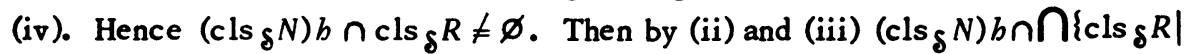
$\left.R \in \Re_{g}\right\} \neq \varnothing$ (since cls ${ }_{\delta} R$ is compact $\left(R \in \Re_{g}\right)$ and (cls $\left.g R \mid R \in \Re_{g}\right)$ has the finite intersection property); i.e. ( $\left(\operatorname{cs}_{\delta} N\right) b \cap L \neq \varnothing$. Again using (ii) and (iii) this implies that $\left.L b \cap L=\left(\bigcap_{\text {cls }} N \mid N \in \Re_{\mathcal{g}}\right\}\right) b \cap L=\bigcap\left\{\left(\operatorname{cls}_{\boldsymbol{g}} N\right) b \cap L \mid N \in \Re_{\boldsymbol{g}}\right\} \neq \varnothing$. Thus $b \in L$ by 3.1. The proof is completed.

3.3 Proposition. Let $\mathfrak{L}$ be a T-subalgebra of $\mathcal{C}(\beta T)$ with $\mathfrak{Q} \subset \mathcal{L}$. Then $H(F, \mathfrak{Q})=\bigcap\left\{\operatorname{cls}_{\mathfrak{Q}} N \mid N \in \Re_{\mathbb{Q}}\right\}$. In particular $H(F, \mathfrak{Q})=\bigcap\left\{\operatorname{cls}_{\tau} N \mid N \in \Re_{\mathbb{Q}}\right\}$. Moreover, $H(F, \mathfrak{Q})$ is a r-closed subgroup of $F$.

Proof. In the preceding discussion set $K=F, \mathcal{S}=\tau$, and $\mathcal{T}=\tau(\mathfrak{l})$. Then (i), (ii), (iii), and (iv) are satisfied (see $[2,11.16,11.17]$ ). Then in this case $L=\bigcap\left\{\operatorname{cls}_{\tau} N \mid N \in \Re_{G}\right\}=H(F, \mathfrak{Q})$ by 3.2. Moreover $L$ is $r$-closed by definition and is a subgroup by 3.1 .

The first part of 3.3 now follows from the fact that $\bigcap\left\{c l s_{\tau} N \mid N \in \pi_{a}\right\} \subset$ $\left.\bigcap_{\{c l s} N \mid N \in \Re_{\mathbb{Q}}\right\} \subset H(F, \mathfrak{Q})$.

4. The action of $F$ on $M$. The semigroup structure on $M$ makes the pair $(F, M)$ into a left transformation group when $F$ is provided with the discrete topology. Since $M$ is compact the set of almost periodic points, $\operatorname{ap}(F, M)$ is not vacuous. Indeed, $\operatorname{ap}(F, M) \cap \overline{F p} \neq \varnothing \quad(p \in M)$.

4.1 Lemma. Let $p, q \in M$ with $p \in \overline{F q}$ and $p=p w$ for some idempotent $W$ in $M$. Then $p \in F q w$.

Proof. Let $\left(a_{n}\right)$ be a net in $F$ such that $a_{n} q$ converges to $p$. Let $W, V \subset T$ be such that $W \in p u$ and $V \in u$ with $V u=V$. Then $[W, V] \cap G$ is a typical neighborhood of $p u$ in the r-topology $[2,11.14 .1]$ where $[W, V]=\{r \mid r \in M, W r \cap V \neq \varnothing\}$.

Let $q v=q$ for $v^{2}=v \in M$. If $W p \cap V v=\varnothing$ then $\varnothing=\varnothing u=W p u \cap V v u=$ $W p u \cap V u=W p u \cap V$. Hence $W p \cap V v \neq \varnothing$, i.e. $p \in[W, V v]$. Since $[W, V v]$ is an open subset of $M$, there exists $n_{0}$ such that $a_{n} q \in[W, V v]$; i.e. $W a_{n} q \cap V v \neq \varnothing$ $\left(n \geq n_{0}\right)$. As above this implies that $W a_{n} q u \cap V \neq \varnothing\left(n \geq n_{0}\right)$. Thus $\alpha_{n} q u$ 
converges to $p u$ in the r-topology. Since $F q u$ is r-closed, this implies that $p u \in F q u$. Then $p=p w=p u w \in F q u w=F q w$.

4.2 Corollary. There exists an idempotent $w \in \operatorname{ap}(F, M) \cap \bar{F}$.

Proof. Since $\bar{F}$ is a closed subset of $M$ invariant under the action of $F$, there exists $p \in \bar{F} \cap \operatorname{ap}(F, M)$. Assume $p w=p$. Then by 4.1 with $q=u, p=a w$ for some $a \in F$. Then $w=a^{-1} p \in \alpha^{-1} \bar{F}=\overline{a^{-1} F}=\bar{F}$. Moreover $w \in \operatorname{ap}(F, M)$ since $\operatorname{ap}(F, M)$ is invariant under $F$.

4.3 Remarks. 1. Let $w^{2}=w \in M$. Then since $w$ is a left identity on $M, F$ and $F w$ act in precisely the same way on $M$. Hence ap $(F, M)=\operatorname{ap}(F w, M) .2$. Again let $w^{2}=w \in M$. Then since $u$ is arbitrary in [2] and what went before, the whole discussion remains valid if $u$ is replaced by $w$. If this is done then $\mathcal{F}, \mathfrak{A}$, and $G$ are replaced by $\mathfrak{F}_{w}, \mathfrak{Q}_{w}$, and $G w$ respectively, and the groups $g\left(\mathcal{F}_{w}\right)$ and $g\left(\mathfrak{Q}_{w}\right)$ now become $F w$ and $A w$. Moreover it is straightforward to verify that the map $\alpha \rightarrow \alpha w:(F, r(\mathfrak{Q})) \rightarrow\left(F w, r\left(\mathfrak{Q}_{w}\right)\right)$ is a bicontinuous isomorphism. Thus $H(F, \mathfrak{Q}) w=H\left(F w, \mathfrak{Q}_{w}\right)$.

For the remainder of this section $w$ will denote an idempotent in $M$ with $w \in \operatorname{ap}(F, M) \cap \bar{F}$. The various rotopologies involved are now assumed on $F w$ rather than $F$.

4.4 Lemma. Let $a \in F$ and $V$ a subset of $T$ with $V \in a w$. Then int $_{\tau} \mathrm{cls}_{\boldsymbol{\tau}}(b(V) \cap F w) \neq \varnothing$.

Proof. Since $a w \in \operatorname{ap}(F, M)=\operatorname{ap}(F w, M)$ and $b(V)$ is a neighborhood of $a w$, $F w b(V) \supset \overline{F w}$. Hence there exists a finite subset $L$ of $F$ with $L w b(V) \supset \overline{F w}$ and 4.4 now follows from the fact that $L w$ is finite.

4.5 Lemma. Let $L \subset F w$ with cls int $_{\tau} L=F w$. Then $\bar{L}=\overline{F w}$.

Proof. Let $\alpha \in F$ and $V \in a w$. Then $\varnothing \neq$ int $_{\tau} \operatorname{cls}_{\tau}(b(V) \cap F w)$ by 4.1. Hence $\varnothing \neq$ int $_{\tau}$ cls $_{\tau}(b(V) \cap F w) \cap$ cls $_{\tau}$ int $_{\tau} L$. Then $\varnothing \neq$ int $_{\tau}$ cls $_{\tau}(b(V) \cap F w) \cap$ int $_{\tau} L$ whence $\varnothing \neq$ cls $_{\tau}(b(V) \cap F w) \cap$ int $_{\tau} L$ and so $\phi \neq b(V) \cap F w \cap$ int $_{\tau} L$. Hence $\varnothing \neq b(V) \cap L$. Thus $a w \in \bar{L}$, whence $\overline{F w} \subset \bar{L}$. The reverse inclusion is obvious.

The main idea of the paper is the combination of 4.5 and 3.3 .

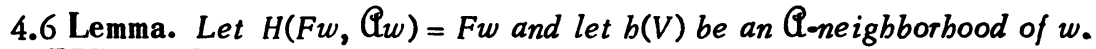
Then $[\overline{V, N] \cap F w}=\overline{F w}(N \in w)$.

Proof. There exist $\epsilon>0$ and finitely many functions $f_{1}, \cdots, f_{n}$ in $\mathfrak{Q}$ such that $\Gamma=\left\{p|p \in M,| f_{i}(p)-f_{i}(\omega) \mid<\epsilon, 1 \leq i \leq n\right\} \subset b(V)$. Since $w p=p(p \in M)$, $\Gamma=\left\{p|p \in M,| f_{i} w(p)-f_{i} w(w) \mid<\epsilon, 1 \leq i \leq n\right\}$. Hence $b(V)$ is an $\mathbb{Q}_{w \text { neighborhood }}$ of $w$. Then $[V, N] \cap F w$ is a $r\left(\mathscr{Q}_{w} w\right.$-neighborhood of $w$ in $F w$. Hence $L=$ int $_{\tau}([V, N] \cap F w) \neq \varnothing$ since int $_{\tau(Q w)}([V, N] \cap F w) \subset L$. 


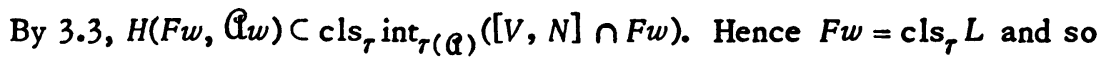
4.6 now follows from 4.5.

Recall that the idempotent $w$ is in $\bar{F}$. Hence $w|\mathcal{F}=u| \mathcal{F}$ and 1.4 follows from

4.7 Proposition. Let $H(F, \mathfrak{Q})=F$ and $P(\mathbb{Q}: \mathcal{F})=\bigcap\left\{\Re_{i} T \cap F(\mathbb{Q}: \mathcal{F}) \mid i \in I\right\}$ where $\left(\Re_{i} \mid i \in I\right)$ is a countable family of indices on $|\mathfrak{Q}|$. Then the set $K=$ $\{p \mid p \in \overline{F w},(p|\mathfrak{Q}, w| \mathfrak{Q}) \in P(\mathfrak{Q}: \mathfrak{F})\}$ is a residual subset of $\overline{F w}$.

Proof. Let $U_{i}$ be a neighborhood of $w \mid \mathfrak{Q}$ such that $U_{i} \times U_{i} \subset \Re_{i}(i \in I)$. Since $|\mathfrak{Q}|$ is minimal $\left(U_{i} \times U_{i}\right) T$ is a neighborhood of the diagonal of $|\mathfrak{Q}| \times|\mathfrak{Q}|$ and so $P(\mathscr{Q}: \mathcal{F})=\bigcap\left\{\left(U_{i} \times U_{i}\right) T \cap R(\mathbb{Q}: \mathcal{F}) \mid i \in I\right\}$.

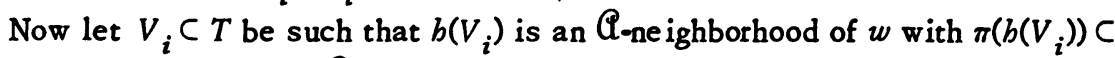
$U_{i}(i \in I)$, where $\pi: M \rightarrow|\mathbb{Q}|$ is the restriction map. (Such exist see $[2,11.13$, 11.14].) Then $P(\mathbb{Q}: \mathcal{F})=\bigcap\left\{\left(\pi b\left(V_{i}\right) \times \pi b\left(V_{i}\right)\right) T \cap R(\mathbb{Q}: \mathcal{F}) \mid i \in I\right\}$.

By 2 of $4.3 H\left(F w, \mathfrak{Q}_{w}\right)=F w$. Hence for all $n,\left[V_{n}, V_{n} w\right] \cap \overline{F w}$ is an open everywhere dense subset of $\overline{F w}$ by 4.6. Thus the intersection, $L$ of all the above sets is a residual subset of $\overline{F w}$.

Let $p \in L$. Then $p\left|\mathfrak{F}_{w}=w\right| \mathfrak{F}_{w}$ since $p \in \bar{F} w$; i.e. $f w p=f w w(f \in \mathcal{F})$. Hence $p|\mathfrak{F}=w| \mathfrak{F}$. Moreover $V_{n} p \cap V_{n} w \neq \varnothing$ implies that $\{p t, w t\} \subset b\left(V_{n}\right)$ for some $t \in T$ and so $\{p, w\} \subset b\left(V_{n}\right) T(n \in I)$. Consequently $(p|\mathfrak{Q}, w| \mathfrak{Q}) \in P(\mathscr{Q}: \mathscr{F})$. The proof is completed.

4.8 Remarks. 1. If $|\mathfrak{Q}|$ is metrizable, then the requirement of $P(\mathfrak{Q}: \mathcal{F})$ in 4.7 is satisfied.

2. Let $f \in a(u)$ be such that the $T$-subalgebra, $[f]$ of $a(u)$ generated by $f$ is separable, and let $\mathfrak{Q}=[\mathcal{F} \cup f]$, the $T$-subalgebra generated by $\mathcal{F} \cup\{f\}$. Then the condition on $P(\mathfrak{Q}: \mathcal{F})$ holds in this case also. To see this let $\left(g_{i}\right)$ be a sequence of functions in $[f]$ which generate $[f]$. Let $N$ denote the positive integers and $£$ the collection of finite subsets of $N$. Set $\eta(L, k)=\left\{(x, y)|x, y \in| \mathbb{Q}|,| g_{i}(x)-\right.$ $\left.g_{i}(y) \mid<1 / k(i \in L)\right\}(L \in \mathfrak{L}, k \in N)$. Then $\eta(L, k)$ is an index on $|\mathfrak{Q}|$ and $P(\mathscr{Q}: \mathcal{F})=$ $\{\eta(L, k) T \cap R(\mathbb{Q}: \mathcal{F}) \mid L \in \mathscr{L}, k \in N\}$.

5. Proof of 2.1. For clarity's sake the proof of 2.1 will be broken up into several parts. Also, throughout this section the following notation will be used: $L=\{\alpha \mid \alpha \in F ;(\alpha|\mathfrak{Q}, u| \mathfrak{Q}) \in Q(\mathfrak{Q}: \mathfrak{F})\}, K=\left\{p \mid p^{2}=p, p\right.$ is in some minimal subset of $\beta T\}$, and $S=\{(x, y) \mid(x v, y v) \in Q(\mathfrak{Q}: \mathcal{F}), v \in K\} \cap R(\mathbb{Q}: \mathcal{F})$.

5.1 Lemma. $Q(\mathfrak{Q}: \mathfrak{F}) \subset S \subset P(\mathfrak{Q}: \mathcal{F}) Q(\mathfrak{Q}: \mathcal{F}) \subset R\left(\mathfrak{U}: \mathfrak{Q} \cap \mathfrak{F}^{\#}\right)$.

Proof. The proof of 14.23 of [2] shows that $Q(\mathfrak{Q}: \mathcal{F}) \subset S$.

Let $(x, y) \in S$, and $v \in K$ with $y v=y$. Since $x|\mathfrak{F}=y| \mathfrak{F}, x|\mathfrak{F}=x v| \mathcal{F}$. Hence $(x, x v) \in P(\mathbb{Q}: \mathfrak{F})$ and so $(x, y)=(x, x v)(x v, y) \in P\left(\mathbb{Q}: \mathfrak{F}^{\prime}\right) Q(\mathfrak{Q}: \mathfrak{F})$.

Since $P(\mathbb{Q}: \mathfrak{F}) \cup Q(\mathfrak{Q}: \mathcal{F}) \subset R\left(\mathfrak{Q}: \mathbb{Q} \cap \mathfrak{F}^{\#}\right)$ and $R\left(\mathbb{Q}: \mathfrak{Q} \cap \mathfrak{F}^{\#}\right)$ is an equivalence relation, $P(\mathbb{Q}: \mathfrak{F}) Q(\mathbb{Q}: \mathcal{F}) \subset R\left(\mathbb{Q}: \mathfrak{Q} \cap \mathfrak{F}^{\#}\right)$. 
5.2 Lemma. If $S$ is an equivalence relation, then $S=R\left(\mathfrak{Q}: \mathfrak{Q} \cap \mathfrak{F}^{\#}\right)$.

Proof. $Q^{2}(\mathfrak{Q}: \mathcal{F}) \subset S^{2}=S \subset P(\mathfrak{Q}: \mathcal{F}) Q(\mathfrak{Q}: \mathcal{F}) \subset Q^{2}(\mathfrak{Q}: \mathfrak{F})$ by 5.1. Since $Q(\mathfrak{Q}: \mathcal{F})$ is closed, so is $Q^{2}(\mathfrak{Q}: \mathcal{F})$. Thus $S$ is a closed invariant equivalence relation containing $Q(\mathfrak{Q}: \mathfrak{F})$ with $S \subset R\left(\mathfrak{Q}: \mathfrak{Q} \cap \mathfrak{F}^{\#}\right)$. Hence $S=R\left(\mathfrak{Q}: \mathfrak{Q} \cap \mathfrak{F}^{\#}\right)$ $[2,14.22]$ since $S$ is invariant (see proof of 14.23 [2]).

\subsection{Lemma. Let $L$ be a group. Then $S$ is an equivalence relation.}

(Thus by $3.3 H(F, \mathfrak{Q})=L$ implies that $S$ is an equivalence relation.)

Proof. Since $S$ is clearly symmetric and reflexive, I shall show only that it is transitive.

Let $(x, y),(y, z) \in S$ and let $\eta \in K$. Choose $p, q, r \in M$ and $\gamma \in K$ such that $p|\mathfrak{Q}=x, q| \mathfrak{Q}=y, r \mid \mathfrak{Q}=z, \gamma \eta=\eta$ and $u y=u$. (Here $u$ is the fixed idempotent. The idempotent $\gamma$ is needed since $\eta$ may not be in the same minimal ideal as $u_{0}$ ) Then $(x y, y \gamma),(y \gamma, z y) \in Q(\mathfrak{Q}: \mathcal{F})$ implies that $\left\{(p \gamma)(q \gamma)^{-1},(q \gamma)(r y)^{-1}\right\} \subset L$. Since $L$ is a group (3.3), $(p \gamma)(r y)^{-1} \in L$. Hence $(x y, z y) \in Q(\mathbb{Q}: \mathfrak{F})$, from which it follows that $(x \eta, z \eta)=(x y \eta, z y \eta) \in Q(\mathfrak{Q}: \mathcal{F})$. The proof is completed.

The following is a restatement of 2.1 .

5.4 Proposition. Statements 1, 2, 3 are pairwise equivalent and they imply statements 4 and 5.

1. $H(F, \mathfrak{Q}) \supset F^{\#}$.

2. $H(F, \mathfrak{Q})=A F^{\#}$.

3. $H(F, \mathbb{Q})=L$.

4. $R\left(\mathbb{Q}: \mathfrak{Q} \cap \mathfrak{F}^{\#}\right)=P(\mathbb{Q}: \mathcal{F}) Q(\mathfrak{Q}: \mathcal{F})$.

5. $R\left(\mathbb{Q}: \mathbb{Q} \cap \mathfrak{F}^{\#}\right)=S$.

Proof. By $[2,14.18] A \subset H(F, \mathfrak{Q}) \subset L \subset A F^{\#}$. This implies that 1 and 2 are equivalent and that 3 follows from 2.

Now 4 follows from 5 by 5.1 . Hence it remains to be shown that 3 implies 5 and 2.

Assume 3. Then $R\left(\mathbb{Q}: \mathbb{Q} \cap \mathfrak{F}^{\#}\right)=S$ by 5.2 and 5.3. (Thus 3 implies 5.) Let $a \in A F^{\#}$. Since $A F^{\#}=g\left(\mathfrak{Q} \cap \mathfrak{F}^{\#}\right),(\alpha|\mathfrak{Q}, u| \mathfrak{Q}) \in R\left(\mathfrak{Q}: \mathfrak{Q} \cap \mathfrak{F}^{\#}\right)=S$. Hence $(\alpha \mid \mathfrak{Q}$, $u \mid \mathfrak{Q}) \in Q(\mathfrak{Q}: \mathcal{F})$ (since $u \in K$ ) and so $a \in L$. Thus $A F^{\#} \subset L=H(F, \mathfrak{Q})$. The proof is completed.

6. The almost periodic points of $R(\mathfrak{Q}: \mathcal{F})$ and the group $H(F, \mathfrak{Q})$. In this section I show that if the almost periodic points are dense in $R(\mathfrak{Q}: \mathfrak{F})$ then the various statements in 5.4 hold. Also in this case $H(F, \mathbb{Q})=A H(F, \tau)=$ $H(F, \tau) A$. Again it will be convenient to let $L$ denote the set $\{a \mid a \in F$, $(\alpha|\mathfrak{Q}, u| \mathfrak{Q}) \in Q(\mathfrak{Q}: \mathfrak{F})\}$. 
6.1 Lemma. Let $\left(a_{n} \mid n \in I\right)$ and $\left(p_{n} \mid n \in I\right)$ be nets on $M$ such that $p_{n} \rightarrow p$, $a_{n} p_{n} \rightarrow q$ and $\left(a_{n}\right) \subset G$. Then $a_{n}^{-1}$ converges to $(p u)(q u)^{-1}$ in the rotopology.

Proof. Let $k \in I$ and $K=\left\{a_{n}^{-1} \mid n \geq k\right\}$. I shall show that $(p u)(q u)^{-1} \in \mathrm{cls}_{\tau} K$. To this end let $f \in \mathcal{C}(\beta T)$ with $f$ real and $a_{n}^{-1} \in K$. Then $\left\langle f, p_{n}\right\rangle=\left\langle f, a_{n}^{-1} a_{n} p_{n}\right\rangle \leq$ $\left\langle f^{K}, a_{n} p_{n}\right\rangle$. Hence $\langle f, p\rangle=\lim \left\langle f, p_{n}\right\rangle \leq \lim \left\langle f^{K}, a_{n} p_{n}\right\rangle=\left\langle f^{K}, q\right\rangle$. This implies that $f p \leq f^{k} q$ for all real $f$ in $\mathcal{C}(\beta T)$. Hence $f(p u)(q u)^{-1} \leq f^{K} u$. The proof is completed.

6.2 Corollary. Let $\left(p_{n} \mid n \in I\right)$ be a net in $M$ and $\left(a_{n} \mid n \in I\right)$ a net in $G$ sucb that $p_{n}|\mathfrak{Q} \rightarrow u| \mathfrak{Q}$ and $a_{n} p_{n}|\mathfrak{Q} \rightarrow a| \mathfrak{Q}$. Then $\left(a_{n}^{-1} \mid n \in I\right)$ converges to $\beta \in A \alpha^{-1} A$ in the r-topology on $G$.

Proof. Let $p_{n} \rightarrow p$ and $a_{n} p_{n} \rightarrow q$. Then $a_{n}^{-1}$ converges to $(p u)(q u)^{-1}$ in the r-topology on $G$. Since $p \mid \mathscr{Q}^{n}=u$ and $q|\mathscr{Q}=\alpha| \mathfrak{Q}, p u \in A$ and $(q u)^{-1} \in \alpha^{-1} A$. The proof is completed.

6.3 Proposition. Let the almost periodic points be dense in $R(\mathbb{Q}: \mathcal{F})$. Then $L=A H(F, \tau)=H(F, \tau) A=H(F, \mathbb{Q})$.

Proof. Since $H(F, \tau)$ is a normal subgroup of $F[2,14.6], A H(F, \tau)=$ $H(F, \tau) A$. Moreover $A H(F, \tau) \subset H(F, \mathfrak{Q})$ since $A \subset H(F, \mathfrak{Q}), r(\mathfrak{Q}) \subset \tau$, and $H(F, \mathfrak{Q})$ is a group.

By $[2,14.18] H(F, \mathfrak{Q}) \subset L$. It thus remains to be shown that $L \subset A H(F, \tau)$.

Let $a \in L$. Then there exist nets $\left(x_{n} \mid n \in I\right)$ and $\left(y_{n} \mid n \in I\right)$ on $|\mathfrak{Q}|$ and elements $t_{n}$ of $T(n \in I)$ such that $\left(x_{n}, y_{n}\right) \in R(\mathbb{Q}: \mathcal{F})(n \in I), x_{n} \rightarrow u\left|\mathfrak{Q}, y_{n} \rightarrow a\right| \mathfrak{Q}$, $x_{n} t_{n} \rightarrow u \mid \mathbb{Q}$, and $y_{n} t_{n} \rightarrow u \mid \mathbb{Q}$.

Since the almost periodic points are dense in $R(\mathbb{Q}: \mathfrak{F})$, we may assume that $\left(x_{n}, y_{n}\right)$ is an almost periodic point of $|\mathfrak{Q}| \times|\mathfrak{Q}|(n \in I)$. This implies that there exist idempotents $v_{n} \in M$ such that $x_{n}=x_{n} v_{n}$ and $y_{n}=y_{n} v_{n}(n \in I)$. Let $p_{n}$, $q_{n} \in M$ with $p_{n} \mid \mathbb{Q}=x_{n}$ and $q_{n} \mid \mathfrak{Q}=y_{n}(n \in I)$. Then we may assume that $p_{n}=p_{n} v_{n}$ and $q_{n}=q_{n} v_{n}(n \in I)$. Since $M v_{n}$ is a group there exist $r_{n} \in M$ with $q_{n}=r_{n} p_{n}=$ $r_{n} u p_{n}$, whence $q_{n}=a_{n} p_{n}$ with $\alpha_{n}=r_{n} u \in G(n \in I)$.

Since $x_{n}\left|\mathfrak{F}=y_{n}\right| \mathfrak{F}, p_{n}\left|\mathfrak{F}=q_{n}\right| \mathfrak{F}=a_{n} q_{n} \mid \mathfrak{F}$ and so $a_{n} \in F$. Moreover $p_{n} \mid \mathfrak{Q} \rightarrow$ $u\left|\mathfrak{Q}, a_{n} p_{n}\right| \mathfrak{Q} \rightarrow a\left|\mathfrak{Q}, p_{n} t_{n}\right| \mathfrak{Q} \rightarrow u \mid \mathbb{Q}$ and $\alpha_{n} p_{n} t_{n}|\mathfrak{Q} \rightarrow u| \mathfrak{Q}$. Now applying 6.2 to the nets $\left(p_{n} \mid n \in I\right)$ and $\left(a_{n} \mid n \in I\right)$ yields that $\alpha_{n}^{-1}$ converges to $\beta \in A a^{-1} A$ in the r-topology, and applying 6.2 to the nets $\left(p_{n} t_{n} \mid n \in I\right)$ and $\left(a_{n} \mid n \in I\right)$ yields that $a_{n}^{-1}$ converges to $\delta \in A u^{-1} A=A$ in the $r$-topology. Since $a_{n}^{-1} \in F(n \in I)$, this implies that $\int \beta^{-1} \in H(F, \tau)$. Hence $a \in A \beta^{-1} A \subset A \mathcal{S}^{-1} H(F, \tau) A \subset A^{2} H(F, \tau) A=$ $A H(F, \tau)$ since $H(F, \tau)$ is normal. The proof is completed.

6.4 Proposition. Let $x \in|\mathfrak{Q}|$ be an F-distal point and let the canonical map 
$\pi:|\mathfrak{Q}| \rightarrow|\mathfrak{F}|$ be open. Then the almost periodic points of $R(\mathfrak{Q}: \mathcal{F})$ are dense in $R(\mathbb{R}: \mathcal{F})$.

Proof. Let $t \in T$ and $y \in|\mathbb{Q}|$ with $\pi(x t)=\pi(y)$. Let $v^{2}=v \in M$ with $y v=y$. Then $x t v|\mathfrak{F}=(x t \mid \mathfrak{F}) v=(y \mid \mathfrak{F}) v=y v| \mathfrak{F}=y|\mathfrak{F}=x t| \mathfrak{F}$. Hence $(x t v, x t) \in P(\mathfrak{I}: \mathfrak{F})$ and so $x t v=x t$. Thus $(x t, y)$ is an almost periodic point of $|\mathfrak{Q}| \times|\mathfrak{Q}|$ which is in $R(\mathfrak{Q}: \mathcal{F})$.

Now let $a, b \in|\mathfrak{Q}|$ with $\pi(a)=\pi(b)$ and let $U$ and $V$ be neighborhoods of $a$ and $b$ respectively. Since $\pi$ is open, $\pi^{-1}(\pi(U) \cap \pi(V)) \cap U$ is a neighborhood of a. Hence there exist $t \in T$ and $y \in V$ with $x t \in U$ and $\pi(x t)=\pi(y)$. The proof is completed.

7. The Veech structure theorem. In this section $(X, T)$ and $(Y, T)$ will denote minimal sets and $\pi: X \rightarrow Y$ a homomorphism. It is also assumed that $X$ is $Y$-point distal; i.e. there exists $x_{0} \in X$ such that if $\pi(x)=\pi\left(x_{0}\right)$ and $x$ is proximal to $x_{0}\left(\left(x, x_{0}\right) \in P(X)\right)$ then $x=x_{0}$.

In order to apply the results of the preceding paragraphs it is necessary to represent $X$ and $Y$ in the form $|\mathfrak{Q}|$ and $|\mathfrak{F}|$ where $\mathcal{Q}$ and $\mathcal{F}$ are $T$-subalgebras of $\mathcal{C}(\beta T)$.

Since $(X, T)$ is minimal, there exists an idempotent $v$ in $M$ such that $x_{0} v=$ $x_{0}$. Since the idempotent $u$ in the preceding section was arbitrary, we may assume that $v=u$.

Now let $\phi:(\beta T, e) \rightarrow\left(X, x_{0}\right)$ and $\Psi:(\beta T, e) \rightarrow\left(Y, y_{0}\right)$ be the canonical homomorphisms where $y_{0}=\pi\left(x_{0}\right)$. Then if $\mathbb{Q}=\phi^{*}(\mathcal{C}(X))$ and $\mathcal{F}=\Psi^{*}(\mathcal{C}(Y)$ ), $\mathcal{F} \subset \mathfrak{Q} \subset a(u)$, there is a commutative diagram

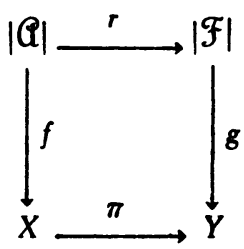

where $r$ is the restriction map and $f$ and $g$ are isomorphisms onto $X$ and $Y$. Moreover $f(u \mid \mathfrak{Q})=x_{0}$.

7.1 Remarks. 1. The assumption that $x_{0}$ is a $Y$-distal point "translates"

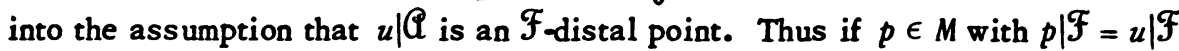
and $(p|\mathfrak{Q}, u| \mathfrak{Q}) \in P(\mathfrak{Q}: \mathcal{F})$, then $p|\mathfrak{Q}=u| \mathfrak{Q}$.

2. The idempotent $w$ of $\S 4$ is in $\bar{F}$. Hence $w|\mathcal{F}=u| \mathcal{F}$. Since $(w|\mathfrak{Q}, u| \mathfrak{Q}) \epsilon$ $P(\mathfrak{Q})$ this implies that $(w|\mathfrak{Q}, u| \mathfrak{Q}) \in P(\mathfrak{Q}: \mathfrak{F})$. Hence $w|\mathfrak{Q}=u| \mathfrak{Q}$.

Although the structure theorem to be proved in this section involves only metric spaces, I would like to prove the basic lemmas in their fullest generality. 
7.2 Definition. Let $R$ and $\mathcal{S}$ be two $T$-subalgebras of $\mathcal{C}(\beta T)$. Then $\mathcal{S}$ is a quasi-separable extension of $R$ if there exists a subset $\mathcal{L}$ of $\delta$ such that $\delta$ is the $T$-subalgebra generated by $R \cup \mathcal{L}$ and such that the $T$-subalgebra, [f] generated by $f$ is separable $(f \in \mathscr{L})$.

7.3 Remarks. 1. If $\mathcal{S}$ is separable (i.e. $|\mathcal{S}|$ is metrizable) and $R \subset S$, then $\delta$ is a quasi-separable extension of $\mathscr{R}$. In this case I shall call $\boldsymbol{S}$ a separable extension of $R$.

2. The $T$-subalgebra $S$ is quasi-separable if and only if it is quasi-separable over the constants, $\mathcal{C}[3,15.1]$.

3. Let $S$ be a quasi-separable extension of $R$ and let $B$ be a $T$-subalgebra with $R \subset B \subset S$. Then $S$ is a quasi-separable extension of $\mathfrak{B}$.

4. Let $S$ be a quasi-separable extension of $R$ and let $B$ be a $T$-subalgebra with $R \subset B$. Then $\mathcal{S} \vee \mathfrak{B}$ is a quasi-separable extension of $\mathfrak{B}$, where $\mathcal{S} \vee \mathfrak{B}$ is the $T$-subalgebra generated by $\mathcal{S} \cup \mathfrak{B}$. (If $\mathscr{R} \cup \mathscr{L}_{\text {generates }} \delta$ then $\mathfrak{B} \cup \mathfrak{Q}$ generates $\delta \vee$ B.)

7.4 Lemma. Let $\mathfrak{Q}$ be a point-distal quasi-separable extension of $\mathcal{F}$ sucb that the restriction map of $|\mathfrak{Q}|$ onto $|\mathfrak{F}|$ is open. Then $\mathfrak{Q} \cap \mathfrak{F}^{\#} \neq \mathcal{F}$ or $\mathfrak{Q}=\mathfrak{F}$.

Proof. Let $\mathfrak{Q} \cap \mathfrak{F}^{\#}=\mathfrak{F}$. I shall prove that $\mathfrak{Q}=\mathfrak{F}$.

First I show that without loss of generality I may assume that $u \mid \mathfrak{Q}_{\text {is }} \mathcal{F}_{\text {-distal. }}$

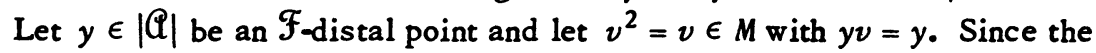
idempotent $u$ was arbitrary we may assume $v=u$. Hence there exists $\alpha \in G$ with $\alpha \mid \mathfrak{Q}=y$.

Now let $(z, u \mid \mathfrak{Q} \alpha) \in P(\mathfrak{Q} \alpha: \mathfrak{F} \alpha)$. Then $z|\mathfrak{F} \alpha=u| \mathfrak{F} \alpha$ and there exists $p \in \beta T$ with $z p=u \mid \mathfrak{Q} a$. Hence $\alpha z|\mathfrak{F}=\alpha u| \mathfrak{F}=\alpha \mid \mathfrak{F}$ and $\alpha z p|\mathfrak{Q}=u \alpha| \mathfrak{Q}=\alpha \mid \mathfrak{Q}$; i.e. $(\alpha z|\mathfrak{Q}, a| \mathfrak{Q}) \in P(\mathfrak{Q}: \mathfrak{F})$. Consequently $\alpha z|\mathfrak{Q}=\alpha| \mathfrak{Q}$; whence $z|\mathfrak{Q} \alpha=u| \mathfrak{Q} \alpha$. Thus $u \mid \mathfrak{Q} a$ is an $\mathcal{F} \alpha$-distal point.

The map $f \rightarrow f a: \mathfrak{Q} \rightarrow \mathfrak{Q} a(f \in \mathfrak{Q})$ is an isomorphism which induces one from $\mathcal{F}$ onto $\mathfrak{F} \alpha$. Thus if there were a nontrivial almost periodic extension of $\mathfrak{F}_{\alpha}$ contained in $\mathbb{Q}_{a}$ there would be a nontrivial almost periodic extension of $\mathfrak{F}$ contained in $\mathfrak{A}$. Hence $\mathfrak{C} a \cap\left(F_{\alpha}\right)^{\#}=F_{a}$. Moreover $\mathfrak{C} \alpha=F_{\alpha}$ if and only if $\mathfrak{Q}=\mathfrak{F}$. Thus as a result of the preceding discussion we may assume that $u \mid \mathfrak{Q}$ is an $\mathfrak{F}_{\text {-distal }}$ point.

I shall complete the proof by showing that the set $N=\{x|x \in| \mathfrak{Q}|, x| \mathfrak{F}=u \mid \mathfrak{F}\}$ consists only of the singleton $\{u \mid \mathfrak{Q}\}$. This suffices because the restriction map being open implies that $R(\mathbb{Q}: \mathcal{F})=\overline{(N \times N) T}$ (see [3, p. 217]).

Let $x \in|\mathfrak{A}|$ with $x|\mathfrak{F}=u| \mathfrak{F}$, and $\mathscr{L} \subset \mathbb{Q}$ be such that $\mathfrak{Q}$ is generated by $\mathfrak{F} \cup \mathcal{Q}$ and $[f]$ is separable $(f \in \mathscr{L})$. Then it suffices to show that $f(x t)=f(u t)(f \in \mathscr{L}$, $t \in T$ ). 
Let $f \in \mathcal{L}$. Set $\mathfrak{B}=[\mathcal{F} \cup f]$, the $T$-subalgebra generated by $\mathcal{F} \cup f$. Let $(y, u \mid \mathfrak{B}) \in P(\mathfrak{B}, \mathfrak{F})$. Then $y|\mathfrak{F}=u| \mathfrak{F}$ and there exists an idempotent $\eta$ with $y=u \eta|\mathfrak{B}=\eta| \mathfrak{B}$. Then $(\eta|\mathfrak{Q}, u| \mathfrak{Q}) \in P(\mathfrak{Q}: \mathcal{F})$, whence $\eta|\mathfrak{Q}=u| \mathfrak{Q}$. Consequently $y=\eta \mid \mathscr{B}$ and so $u \mid \mathfrak{B}$ is an $\mathcal{F}$-distal point.

Since $\mathfrak{Q} \cap \mathfrak{F}^{\#}=\mathcal{F}, F=g(\mathfrak{F})=g\left(\mathbb{Q} \cap \mathfrak{F}^{\#}\right)=A F^{\#}$, whence by $6.4,6.3$, and 5.4 $H(F, \mathfrak{Q})=F$. Since $\mathfrak{B} \subset \mathbb{Q}, r(\mathfrak{B}) \subset r(\mathfrak{Q})$ and so $H(F, \mathfrak{B})=F$.

By 2 of 4.8 and 4.7 the set $K=\{p \mid p \in \overline{F w},(p|\mathfrak{B}, w| \mathfrak{B}) \in P(\mathfrak{B}: \mathcal{F})\}$ is a residual subset of $\overline{F w}$, where $w$ is an idempotent in $M$ with $w|\mathfrak{F}=u| \mathfrak{F}$. Since $(w|\mathfrak{B}, u| \mathfrak{B}) \epsilon$ $P(\Re, \mathcal{F})$ and $u \mid \mathfrak{B}$ is an $\mathcal{F}$-distal point, $w|\mathfrak{B}=u| \mathfrak{B}$. Similarly $p|\mathfrak{B}=u| \mathfrak{B}(p \in K)$. Since $K$ is dense in $\overline{F w}$ this implies that $p|\mathscr{B}=u| \mathfrak{B}(p \in \overline{F w})$. Thus in particular $a w|\mathfrak{B}=u| \mathfrak{B}$ and so $\alpha|\mathfrak{B}=u| \mathfrak{B}(\alpha \in F)$, i.e. $F=B=g(\mathfrak{B})$.

Now let $x v=x$ for some idempotent $v$ in M. (Recall that $x$ is an arbitrary element of $|\mathfrak{Q}|$ with $x|\mathfrak{F}=u| \mathfrak{F}$.) Then $u|\mathfrak{F}=x| \mathfrak{F}=x v \mid \mathfrak{F}=(x \mid \mathfrak{F}) v=(u \mid \mathfrak{F}) v=$ $u v|\mathfrak{F}=v| \mathfrak{F}$ and, since $(u|\mathfrak{B}, v| \mathfrak{B}) \in P(\mathfrak{B}), u|\mathfrak{B}=v| \mathfrak{B}$. Moreover $x u|\mathfrak{F}=u| \mathfrak{F}$. Hence if $p \in M$ with $p \mid \mathfrak{Q}=x$, then $p u \in F=B$. Thus $p u|\mathfrak{B}=u| \mathfrak{B}$, whence $x \mid \mathfrak{B}=$ $p|\mathfrak{B}=x v| \mathfrak{B}=p v|\mathfrak{B}=u v| \mathfrak{B}=v|\mathfrak{B}=u| \mathfrak{B}$. Consequently $f(x t)=f(u t)(t \in T)$. The proof is completed.

7.5 Lemma. Let $\mathfrak{Q}$ be a separable extension of $\mathcal{F}$ and let $u \mid \mathfrak{Q}$ be an $\mathcal{F}$-distal point. Then there exist separable almost automorpbic extensions $\mathcal{L}$ and $\mathbb{B}$ of $\mathfrak{Q}$ and $\mathcal{F}$ respectively such that the restriction map of $|\mathfrak{Q}|$ onto $|\mathfrak{B}|$ is open and such that $u \mid £$ is a B-distal point (see Definition 1.2).

Proof. By Theorem 3.1 of [3] and the remarks at the beginning of this section there exist separable $T$-subalgebras $\mathcal{K}$ and $\mathfrak{B}$ of $a(u)$ which are almost automorphic extensions of $\mathfrak{Q}$ and $\mathfrak{F}$ respectively and such that the restriction map $r:|\mathcal{K}| \rightarrow|\mathfrak{B}|$ is open.

Set $\mathfrak{Q}=\mathbb{Q} \vee \mathfrak{B}$, the $T$-subalgebra generated by $\mathfrak{Q} \cup \mathfrak{B}$. Then $\mathfrak{L} \subset \mathcal{K}$ and so $\mathscr{L}$ is an almost automorphic extension of $\mathfrak{Q}$. Also the commutative diagram

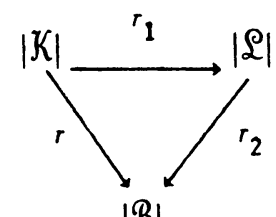

$|B|$

where all the maps involved are restrictions shows that $r_{2}$ is open.

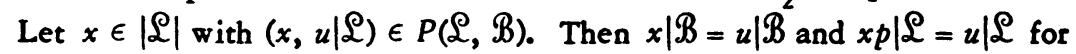
some idempotent $p \in \beta T$. Hence $x|\mathcal{F}=u| \mathfrak{F}$ and $x p|\mathfrak{Q}=u| \mathfrak{Q}$; i.e. $(x|\mathfrak{Q}, u| \mathfrak{Q}) \epsilon$ $P(\mathfrak{Q}: \mathcal{F}$ ). Consequently $x|\mathfrak{Q}=u| \mathfrak{Q}$, whence $x|\mathfrak{Q}=u| \mathfrak{Q}$ (recall $\mathfrak{L}=\mathfrak{Q} \vee \mathfrak{B}$ ). Thus $u \mid \mathfrak{Q}$ is a $B$-distal point. 
The following is the algebraic version of the Veech structure theorem as extended by Bronstein.

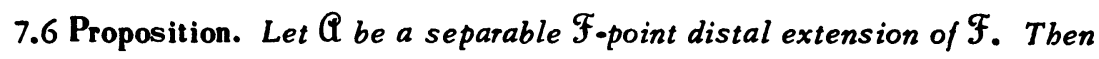
there exist an ordinal $\nu$ and a family $\left(\mathscr{B}_{a} \mid a \leq \nu\right)$ of separable $T$-subalgebras such that (i) $\mathfrak{B}_{0}=\mathcal{F}$, (ii) $\mathfrak{B}_{\nu}$ is an almost automorphic extension of $\mathfrak{A}$, (iii) $\mathfrak{B}_{a+1}$ is either an almost automorphic extension of $\mathfrak{B}_{a}$ or a nontrivial almost periodic extension of $\mathfrak{B}_{a}(a+1 \leq \nu)$, (iv) $\mathfrak{B}_{a}=\overline{\cup\left\{\mathfrak{B}_{\gamma} \mid \gamma<a\right\}}$ for all limit ordinals $a \leq \nu$. Moreover, the set $\{a \mid a \leq \nu\}$ is countable.

Proof. Without loss of generality as in the proof of $7.4 \mathrm{I}$ may assume that $u \mid \mathfrak{Q}$ is an $\mathcal{F}_{\text {-distal point. }}$

Let $\Omega$ be the first uncountable ordinal. I shall construct two families $\left(\Re_{\alpha} \mid a \leq \Omega\right)$ and $\left(\varrho_{\alpha} \mid a \leq \Omega\right)$ of $T$-subalgebras such that:

(a) the family $\left(\mathscr{B}_{a} \mid a \leq \Omega\right)$ satisfies conditions (i), (iii), and (iv) with $\nu$ replaced by $\Omega$,

(b) $\mathscr{L}_{a} \subset \mathscr{L}_{\beta}(a \leq \beta \leq \Omega)$,

(c) $\mathscr{L}_{\alpha}$ is a separable almost automorphic extension of $\mathfrak{Q}(\alpha<\Omega)$,

(d) $\mathfrak{B}_{a} \subset \mathscr{L}_{a}$ and $u \mid \mathfrak{Q}_{a}$ is a $\mathfrak{B}_{a}$-distal point $(\alpha \leq \Omega)$.

Set $\mathfrak{B}_{0}=\mathfrak{F}, \mathfrak{L}_{0}=\mathfrak{Q}$ and assume $\mathfrak{B}_{a}$ and $\mathfrak{Q}_{\alpha}$ defined for all $a<\gamma<\Omega$ such that the families $\left(\mathscr{B}_{a} \mid a<\gamma\right)$ and $\left(\mathscr{C}_{a} \mid a<\gamma\right)$ satisfy (a), (b), (c), and (d). If $\gamma$ is a limit ordinal, set $\mathscr{B}_{\gamma}=\bar{U}\left\{\mathscr{B}_{a} \mid \alpha<\gamma\right\}$ and $\mathfrak{L}_{\gamma}=\bar{U}\left\{\mathfrak{Q}_{a} \mid a<\gamma\right\}$. Then it is easy to verify that the families $\left(\mathfrak{B}_{a} \mid a \leq \gamma\right)$ and $\left(\bigodot_{a} \mid \alpha \leq \gamma\right)$ satisfy (a), (b), and (d). With regards to (c), observe that since $\gamma<\Omega,\{a \mid a \leq y\}$ is countable whence $\mathscr{L}_{\gamma}$ is separable. Moreover the set $A_{a}$ of points $x$ in $\left|\mathfrak{Q}{ }_{a}\right|$ such that the fiber over $x \mid \mathfrak{Q}$ is a singleton is a residual subset of $\left|\mathfrak{C}_{a}\right|(\alpha \leq \gamma)$. Hence $\mathfrak{L}_{\gamma}$ is an almost automorphic extension of $\mathbb{Q}$ (see also [3, p. 223]).

Let $y=a+1$. If the restriction map of $\left|\mathscr{C}_{a}\right|$ onto $\left|B_{a}\right|$ is open then by 7.4 either (A) $\mathfrak{L}_{a}=\mathfrak{B}_{a}$, or (B) there exists a nontrivial almost periodic extension $K$ of $\mathfrak{B}_{a}$ with $\mathfrak{B}_{a} \subset \mathcal{K} \subset \mathfrak{L}_{a}$. In case (A) set $\mathfrak{B}_{a+1}=\mathfrak{L}_{a}=\mathfrak{L}_{a+1}$ and in case (B) set $\mathfrak{B}_{a+1}=\mathcal{K}$ and $\mathscr{L}_{a+1}=\mathscr{L}_{a}$. Then again one verifies directly that (a), (b), (c), and (d) hold for the families $\left(\mathfrak{B}_{\alpha} \mid a \leq \gamma\right)$ and $\left(\Re_{a} \mid a \leq \gamma\right)$.

If the restriction map of $\left|\mathfrak{Q}_{a}\right|$ onto $\left|\mathfrak{B}_{a}\right|$ is not open, then by 7.5 there exist separable almost automorphic extensions $\mathfrak{L}_{a+1}$ and $\mathfrak{B}_{a+1}$ of $\mathfrak{L}_{a}$ and $\mathfrak{B}_{a}$ respectively such that the restriction map of $\left|\mathfrak{Q}_{a+1}\right|$ onto $\left|\mathfrak{B}_{a+1}\right|$ is open and $u \mid \mathfrak{Q}_{a+1}$ is a $\mathfrak{B}_{a+1}$-distal point.

For some $\nu<\Omega$ we must have $\mathscr{L}_{\nu}=\mathfrak{B}_{\nu^{*}}$ (See Lemmas 4.2 and 4.3 of [3], and note that no use is made of the assumption that there is a fiber with a dense set of distal points in order to prove that there can be at most countably many distinct $\mathfrak{L}_{a}$ 's.) The proof is completed. 
As Veech remarks $[3$, p. 224] an induction using 7.6 yields:

7.7 Proposition. Let $\mathfrak{Q}$ be a separable point-distal extension of $\mathcal{F}$. Then the

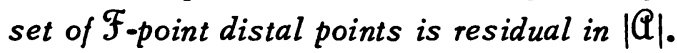

8. An extension of the structure theorem. In this section I would like to discuss what is involved in using 7.2 to extend the Veech structure theorem to the quasi-separable case. Proposition 7.4 shows that if $X$ is a quasi-separable $Y$ point distal extension of $Y$ and $\pi: X \rightarrow Y$ is open then either $X=Y$ or a nontrivial almost periodic extension of $Y$ can be "interpolated" between $X$ and $Y$. The trouble arises when $\pi$ is not open. In the metric case Veech overcomes this difficulty by constructing almost automorphic extensions $X^{*}$ and $Y^{*}$ of $X$ and $Y$ respectively and homomorphisms $\pi^{*}: X^{*} \rightarrow Y^{*}, p: X^{*} \rightarrow X$, and $q: Y^{*} \rightarrow Y$ such that the diagram

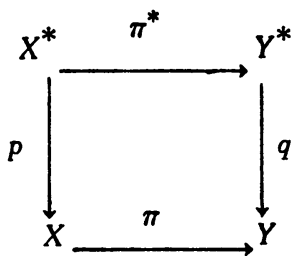

is commutative, $X^{*}$ is a metrizable $Y^{*}$-point distal extension of $Y^{*}$ and $\pi^{*}$ is open. In the general case I shall show that one can construct $X^{*}$ and $Y^{*}$ as above with $\pi^{*}$ open. However, the extensions $X^{*}$ and $Y^{*}$ of $X$ and $Y$ are no longer almost automorphic ones but merely proximal ones.

Recall that $\pi: X \rightarrow Y$ is proximal if $x_{1}$ and $x_{2}$ are proximal whenever $\pi\left(x_{1}\right)=$ $\pi\left(x_{2}\right)$. In terms of algebras, $\mathcal{Q}$ is a proximal extension of $\mathcal{K}$ if $R(£: \mathcal{K}) \subset P(£: \mathcal{K})$ and this is true if and only if $g(£)=g(\mathcal{K})$ (see $[2,13.6]$ ).

Thus in order to generalize Veech's theorem to the quasi-separable case I shall show that $a(F) \vee \mathcal{Q}$ is an open extension of $a(F)$, where for any r-closed subgroup $K$ of $G a(K)=\{f \mid f \in \mathcal{C}(\beta T)$ and $f \alpha=f(\alpha \in K)\}$. The idea of the proof is due to H. Furstenberg.

For the remainder of this section $X$ and $Y$ will denote minimal sets and $\pi: X \rightarrow Y$ a homomorphism. The collection of nonempty closed subsets of $X$ provided with the uniform structure induced by the uniformity on $X$ will be denoted $2^{X}$. Then $2^{X}$ is a compact Hausdorff space on which $T$ acts via the map $(K, t) \rightarrow$ $K t: 2^{X} \times T \rightarrow 2^{X}$ where $K t=\{k t \mid k \in K\}\left(K \in 2^{X}, t \in T\right)$. Let $W=\operatorname{cls}\left\{\pi^{-1}(y) \mid\right.$ $y \in Y\}$. Then $W$ is a closed invariant subset of $2^{X}$ and there exists a homomorphism $\Psi: W \rightarrow Y$ such that $\Psi(K)=y$ where $K \subset \pi^{-1}(y)(K \in W)$.

8.1 Lemma. Let $x \in X$. Then there exists $K \in \overline{\pi^{-1} \pi(x) T} \subset W$ such that $x \in K$ and $\overline{K T}$ is a minimal subset of $W$. 
Proof. Since $x$ is an almost periodic point of $X$ there exists an idempotent $v \in M$ with $x v=x$. Let $\left(t_{n}\right)$ be a net converging to $v$. Then $\left(\pi^{-1} \pi(x)\right) t_{n}$ converges to some $K \in W$. Since $K v=K, \overline{K T}$ is minimal. (Here $K$ is viewed as a point of the transformation group $\left(2^{X}, T\right)$.) Also $x \in \pi^{-1} \pi(x)$ and $x t_{n} \rightarrow x$ implies that $x \in K$. The proof is completed.

8.2 Lemma. Let $Z$ be a minimal subset of $\overline{\pi^{-1} \pi(x) T}$ for some $x \in X$. Then $(Z, \Psi)$ is a proximal extension of $Y$.

Proof. It suffices to show that if $K$ and $L$ are in $Z$ with $\Psi(K)=x=\Psi(L)$, then $K$ and $L$ are proximal. To show this let $N \in Z$ with $\Psi(N)=x$ and such that if $R \in Z$ with $R \subset N$ and $\Psi(R)=x$, then $R=N$. (A simple application of Zorn's lemma shows that such an $N$ exists.)

Since $N \in \overline{\left(\pi^{-1} \pi(x)\right) T}$, there exists a net $\left(t_{n}\right)$ such that $\left(\pi^{-1} \pi(x)\right) t_{n} \rightarrow N$. We may assume $K t_{n} \rightarrow R$ and $L t_{n} \rightarrow S$. Then $R, S \in \overline{\left(\pi^{-1} \pi(x)\right) T}$ and, since $K \cup L \subset \pi^{-1} \pi(x), R \cup S \subset N$. Hence $R=N=S$. The proof is completed.

8.3 Proposition (Furstenberg). Let $Y$ be such that it admits no proper minimal proximal extensions. Then $\pi: X \rightarrow Y$ is open.

Proof. Let $U$ be an open subset of $X$. If $\pi(U)$ were not open there would exist $x \in U$ and a net $\left(y_{n} \mid n \in I\right)$ on $Y$ such that $\left(y_{n} \mid n \in I\right) \rightarrow y=\pi(x)$ and $U \cap \pi^{-1}\left(y_{n}\right)=\varnothing(n \in I)$.

Let $K \subset \pi^{-1} \pi(x)$ be such that $x \in K$ and $\overline{K T}=Z$ is a minimal subset of $\overline{\left(\pi^{-1} \pi(x)\right) T}$. Then $(Z, \Psi)$ is a proximal extension of $Y$ and so $\Psi$ is one-one.

Let $K_{n} \in Z$ be such that $\Psi\left(K_{n}\right)=y_{n}(n \in I)$. We may assume that $\left(K_{n} \mid n \in I\right)$ $\rightarrow L \in Z$. Then $\Psi(L)=\lim \Psi\left(K_{n}\right)=\lim y_{n}=y=\Psi(K)$. Hence $L=K$, and so $\varnothing \neq K_{n} \cap U \subset \pi^{-1}\left(y_{n}\right) \cap U$ for some $n \in I$ (recall that $x \in K$ and $U$ is a neighborhood of $x$ ). This is a contradiction, and so $\pi(U)$ is open.

8.4 Corollary. Let $L$ be a r-closed subgroup of $G$ and let $\mathcal{K}$ be a $T$-subalgebra of $a(u)$ with $a(L) \subset K$. Then the restriction map of $|\mathcal{K}|$ onto $|a(L)|$ is open.

Proof. The transformation group $|a(L)|$ has no proper proximal extensions.

8.5 Proposition. Let $\mathfrak{Q}$ be a point-distal quasi-separable extension of $\mathfrak{F}$. Then there exists a family of T-subalgebras $\left(\mathscr{B}_{a} \mid \alpha \leq \nu\right)$ sucb that (i) $\mathfrak{B}_{0}=\mathfrak{F}$, (ii) $\mathfrak{B}_{a}$ is a proximal extension of $\mathfrak{A}$, (iii) $\mathfrak{B}_{a+1}$ is either an almost periodic or a proximal extension of $\mathfrak{B}_{a}(\alpha+1 \leq \nu)$, (iv) $\mathfrak{B}_{a}=\bar{U}\left\{\mathfrak{B}_{a} \mid \gamma<\alpha\right\}$ for all limit ordinals $a \leq \nu$.

Proof. Let $\delta$ be an ordinal with cardinal greater than the cardinal of $F$. Set $\mathfrak{B}_{0}=\mathcal{F}, \mathscr{\complement}_{0}=\mathfrak{A}$, and assume $\left(\mathfrak{B}_{a} \mid a<\gamma\right)$ and $\left(\aleph_{\alpha} \mid a<\gamma\right)$ defined such that 
(a) $\left(\Re_{a} \mid a \leq \delta\right)$ satisfies (i), (iii) and (iv) with $\nu$ replaced by $\delta(a<\gamma)$,

(b) $\mathfrak{L}_{a}$ is a quasi-separable extension of $\mathfrak{B}_{a}(a<\gamma)$,

(c) $u \mid \mathbb{Q}_{a}$ is a $\Re_{a}$-distal point $(a<\gamma)$,

(d) $g\left(\varrho_{a}\right)=A(a<\gamma)$. (As in the proof of $7.6 \mathrm{I}$ assume $u \mid \mathbb{Q}$ is an $\mathcal{F}_{\text {-distal }}$ point.)

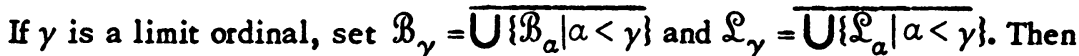
it is immediate that $\left(\mathscr{B}_{\alpha} \mid a \leq \gamma\right)$ and $\left(\varrho_{\alpha} \mid a \leq \gamma\right)$ satisfy (a), (b), (c), and (d).

Let $\gamma=\beta+1$. Let $\mathscr{B}_{\beta}=g\left(\mathscr{B}_{\beta}\right)$. If $\mathfrak{B}_{\beta} \neq a\left(\mathscr{B}_{\beta}\right)$, set $\mathfrak{B}_{\beta+1}=a\left(B_{\beta}\right)$ and $\mathscr{L}_{\beta+1}=a\left(B_{\beta}\right) \vee \mathscr{L}_{\beta}$ Then $g\left(\mathfrak{B}_{\beta+1}\right)=B_{\beta}$ and $g\left(\mathfrak{\complement}_{\beta+1}\right)=B_{\beta} \cap g\left(\mathscr{C}_{\beta}\right)=B_{\beta} \cap A=A$ (since $\mathfrak{B}_{\beta} \subset \mathscr{L}_{\beta}$ implies that $A=g\left(\mathfrak{Q}_{\beta}\right) \subset B_{\beta}$ ). Hence in this case the families $\left(B_{a} \mid \alpha \leq \gamma\right)$ and $\left(\varrho_{a} \mid a \leq \gamma\right)$ satisfy (a), (b), (c) and (d).

Let $\gamma=\beta+1$ and $B_{\beta}=a\left(B_{\beta}\right)$. Then $\mathscr{L}_{\beta}$ is an open separable point-distal extension of $\mathfrak{B}_{\beta}$. By 7.2 either there exists a nontrivial almost periodic extension $\mathcal{K}$ of $\mathfrak{B}_{\beta}$ with $\mathcal{K} \subset \mathscr{L}_{\beta}$ or $\mathscr{L}_{\beta}=\mathscr{B}_{\beta}$ In the first case set $\mathfrak{B}_{\beta+1}=\mathcal{K}$ and $\mathfrak{L}_{\beta_{+1}}=\mathscr{L}_{\beta}$. In the second case set $\mathfrak{B}_{\beta+1}=\mathscr{B}_{\beta}$ and $\mathscr{L}_{\beta+1}=\mathscr{L}_{\beta}$.

Thus the construction need not be carried beyond the point where $B_{\beta}=A$ 。 Now at each stage $A \subset B_{\beta} \subset F$. If $B_{\beta} \neq A$ then $B_{\beta+2} \subsetneq B_{\beta}$. Hence there is an ordinal $\nu \leq \delta$ such that $B_{\nu}=\mathfrak{L}_{\nu}$. The proof is completed.

\section{REFERENCES}

1. I. Bronštěn, $A$ theorem on the structure of almost distal expansions of minimal sets, Math. Issled. 6 (1971), vyp. 2 (20), 22-32, 157. (Russian) MR 44 \#7532.

2. R. Ellis, Lectures on topological dynamics, Benjamin, New York, 1969. MR 42 \#2463.

3. W. A. Veech, Point-distal flows, Amer. J. Math. 92 (1970), 205-242. MR 42 \#2462.

SCHOOL OF MATHEMATICS, UNIVERSITY OF MINNESOTA, MINNEAPOLIS, MINNESOTA 55455 